\title{
Entanglement and symmetry effects in the transition to the Schrödinger cat regime
}

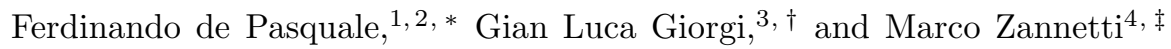 \\ ${ }^{1}$ Dipartimento di Fisica, Università di Roma La Sapienza, Piazzale A. Moro 2, 00185 Roma, Italy \\ ${ }^{2}$ CNR-INFM Center for Statistical Mechanics and Complexity \\ ${ }^{3}$ Institute for Cross-Disciplinary Physics and Complex Systems, IFISC (CSIC-UIB), \\ Campus Universitat Illes Balears, E-07122 Palma de Mallorca, Spain \\ ${ }^{4}$ Dipartimento di Matematica e Informatica, Università di Salerno, \\ via Ponte don Melillo, 84084 Fisciano (SA), Italy
}

\begin{abstract}
We study two-spin entanglement and order parameter fluctuations as a function of the system size in the XY model in a transverse field and in the isotropic XXX model. Both models are characterized by the occurrence of ground state degeneracy also when systems of finite size are considered. This is always true for the XXX model, but only at the factorizing field for the XY model. We study the size dependence of symmetric states, which, in the presence of degeneracy, can be expanded as a linear combination of broken symmetry states. We show that, while the XY model looses its quantum superposition content exponentially with the size $N$, a decrease of the order of $1 / N$ is observed when the XXX model is considered. The emergence of two qualitatively different regimes is directly related to the difference in the symmetry of the models.
\end{abstract}

PACS numbers: 05.30.-d, 03.65.Ud, 64.70.Tg

\section{INTRODUCTION}

The transition between the microscopic and macroscopic worlds is a fundamental issue in quantum theory both from the point of view of foundations of physics and of the application to quantum computation [1, 2].

Spontaneous symmetry breaking (SSB) indicates a situation where, given a symmetry of the Hamiltonian, there are eigenstates which are not invariant under the action of this symmetry, unless a term is added which explicitly breaks the symmetry. Usually, when the control parameter reaches a critical value, the lowest energy eigenstate keeping the Hamiltonian symmetry is no longer stable in the presence of infinitely small perturbations, and new stable solutions appear which are not symmetric. SSB leads naturally to a degenerate manifold of ground states.

Symmetry breaking usually occurs in the thermodynamic limit, when superselection destroys quantum coherence. Important exceptions are the XXX Heisenberg model and the XY model in a transverse magnetic field at the particular value of the field where ground state factorization occurs [3]. In these cases, ground state degeneracy occurs for any size of the system, and it is therefore possible to explicitly study the transition from quantum to classical behavior. How entanglement is affected, in the thermodynamic limit, by the presence of a term which explicitly breaks the symmetry, ha been discussed by Syljuåsen [4] and by Osterloh et al. [5]. Here, we face the problem from another point of view, starting from small systems, and then increasing the size until the thermodynamic limit is reached.

The XY model in a transverse field has been introduced in the early sixties and solved by Katsura [6], by means of the Jordan-Wigner transformation, formerly introduced by Lieb, Schultz, and Mattis [7]. Subsequently, the correlation functions were investigated in great detail by Barouch and MacCoy [8], who found the existence of a second critical value of the transverse field separating qualitatively different behaviors of the correlation functions. Later on, Kurmann, Thomas and Muller [3] discovered ground state factorization for a large class of spin models. In the particular case of the XY model, the field at which factorization occurs is exactly the critical field of Barouch and MacCoy. Recent interest has been devoted to the study of entanglement properties of many-body systems undergoing a quantum phase transition [9-12]. As shown in Ref. [13], the critical point turns out to separate two regions with qualitatively different bipartite entanglement. It has been shown in Ref. [14] that, in the vicinity of the factorizing field, the range of concurrence diverges, and that such divergence corresponds the appearance of a characteristic length scale in the system. Recently, the conditions for the existence of the factorizing field for models with long-range interaction have been determined [15], and the study of this effect has been extended to dimerized chains [16].

\footnotetext{
*Electronic address: ferdinando.depasquale@roma1.infn.it

${ }^{\dagger}$ Electronic address: gianluca@ifisc.uib-csic.es

${ }^{\ddagger}$ Electronic address: zannetti@sa.infn.it
} 
The two systems we wish to investigate belong to different classes of symmetry. While the XXX Heisenberg model has the $\mathrm{SU}(2)$ continuous symmetry, i.e. the Hamiltonian commutes with the total spin along any possible direction, the XY model is invariant under parity transformations and possesses the discrete $Z_{2}$ symmetry.

It is commonly accepted that purely quantum effects are not observable on the macroscopic scale, except for superconductivity, superfluidity. On the other hand, quantifying entanglement (perhaps the most genuine manifestation of quantum properties) as a function of the system size represents a fundamental issue [17]. Here, we wish to investigate in detail the two-spin entanglement dependence on the total number of spins for these models. In particular, we shall derive the difference in the size effects due to the difference in the system symmetry. To be more specific, in the case of a discrete symmetry there is an exponential entanglement decrease, while with a continuous symmetry entanglement shrinks linearly with the growth of the system size.

Since the XY model has been studied through the last four decades, and results are scattered over a vast literature, for convenience we shall give here a brief survey of the main results, with the primary aim of focusing on the existence of the factorizing field and its independence from the system size.

The paper is organized as follows. In Sec. II the XY model in a transverse field is discussed. We give special emphasis to the finite size solution with the scope of enlightening the emergence of the factorizing field as a sizeindependent degeneracy point. Furthermore, by means of the finite size picture, we are able to explain in a simple way the appearance of spontaneous symmetry breaking in the thermodynamic limit. In Sec. III we describe briefly the structure of the ground state for the isotropic Heisenberg (XXX) model. Even if, for any finite number of spins the ground state manifold has finite dimension, an over-complete set of states can be introduced that allows to study the microscopic-to-macroscopic transition. In Sec. IV we derive the value of the concurrence for pairs of spins and the order parameter fluctuation in a superposition state as a function of the size systems both for the XY model and the XXX model. Finally, in Sec. V] results are discussed. In particular, we will focus on the influence of the symmetry in the different behaviors.

\section{XY MODEL}

Let us consider a chain of $N$ spins

$$
H=\sum_{l}\left[J \frac{(1+\gamma)}{2} \sigma_{l}^{x} \sigma_{l+1}^{x}+J \frac{(1-\gamma)}{2} \sigma_{l}^{y} \sigma_{l+1}^{y}+h \sigma_{l}^{z}\right],
$$

where $\sigma^{\epsilon}$ are the three Pauli matrices $(\epsilon=x, y, z)$, and periodic boundary conditions $\left(\sigma_{N+1}^{\epsilon}=\sigma_{1}^{\epsilon}\right)$ are assumed. In the following we will assume $J=-1$ (ferromagnetic coupling). The above Hamiltonian is invariant under the $Z_{2}$ group of the rotations by $\pi$ about the $z$ axis, since it commutes with the parity operator $P=\prod_{l} \sigma_{l}^{z}$. Due to this symmetry, eigenstates are classified depending on parity eigenvalue. This system is known to undergo a quantum phase transition at the critical point $h_{c}=1$. Below this value, in the thermodynamic limit, spontaneous magnetization along the $x$ axis appears.

Since the work of Ref. [7], the Jordan-Wigner transformation, defined through $\sigma_{l}^{z}=1-2 c_{l}^{\dagger} c_{l}, \sigma_{l}^{+}=$ $\prod_{j<l}\left(1-2 c_{l}^{\dagger} c_{l}\right) c_{l}, \sigma_{l}^{-}=\prod_{j<l}\left(1-2 c_{l}^{\dagger} c_{l}\right) c_{l}^{\dagger}$ is introduced to map spins in spinless fermions. The transformed Hamiltonian is $H=H_{0}-P H_{1}$ with

$$
\begin{aligned}
& H_{0}=-\sum_{l=1}^{N-1}\left[\left(c_{l}^{\dagger} c_{l+1}-c_{l} c_{l+1}^{\dagger}\right)+\gamma\left(c_{l}^{\dagger} c_{l+1}^{\dagger}-c_{l} c_{l+1}\right)-h\left(1-2 c_{l}^{\dagger} c_{l}\right)\right], \\
& H_{1}=-\left[\left(c_{N}^{\dagger} c_{1}-c_{N} c_{1}^{\dagger}\right)+\gamma\left(c_{N}^{\dagger} c_{1}^{\dagger}-c_{N} c_{1}\right)\right] .
\end{aligned}
$$

Since $[H, P]=0$, all eigenstates of $H$ have definite parity, and we can proceed to a separate diagonalization of $H$ in the two subspaces labelled by to $P= \pm 1$. Then, the complete set of eigenvectors of $H$ will be given by the even eigenstates of $H^{+}=H_{0}-H_{1}$ and the odd eigenstates of $H^{-}=H_{0}+H_{1}$. Both for $H^{+}$and $H^{-}$the diagonalization is obtained by first carrying out the space Fourier transform

$$
c_{k}=\frac{1}{\sqrt{N}} \sum_{k} e^{-i \frac{2 \pi}{N} k l} c_{l}
$$

where $k=0,1, \ldots, N-1$ in $H^{-}$, and $k=1 / 2,3 / 2, \ldots, N-1 / 2$ in $H^{+}$, and then making the Bogoliubov transformation

$$
c_{k}=\cos \vartheta_{k} \eta_{k}+i \sin \vartheta_{k} \eta_{-k}^{\dagger}
$$


with $\vartheta_{k}=-\vartheta_{-k}$. Here, $\eta_{-k}$ stands for $\eta_{N-k}$. The diagonalization condition implies for $\vartheta_{k}$

$$
\tan 2 \vartheta_{k}=-\frac{\gamma \sin k}{h-\cos k}
$$

Eventually, we end up with the quasi-particle Hamiltonians

$$
\begin{aligned}
H^{+} & =\sum_{k=1 / 2}^{N-1 / 2} \Lambda_{k}\left(\eta_{k}^{\dagger} \eta_{k}-\frac{1}{2}\right), \\
H^{-} & =\sum_{k=0}^{N-1} \Lambda_{k}\left(\eta_{k}^{\dagger} \eta_{k}-\frac{1}{2}\right),
\end{aligned}
$$

where the eigenvalues are given by

$$
\Lambda_{k}=2 \sqrt{\left(h-\cos \frac{2 \pi}{N} k\right)^{2}+\gamma^{2} \sin ^{2} \frac{2 \pi}{N} k}
$$

The ground states of $H^{+}$and $H^{-}$are the corresponding vacuum states with eigenvalues $E_{0}^{+}=-\sum_{k=1 / 2}^{N-1 / 2} \Lambda_{k}$ and $E_{0}^{-}=-\sum_{k=0}^{N-1} \Lambda_{k}$.

The vacuum in the generic $k$ mode is determined by $\eta_{k}\left|0^{ \pm}\right\rangle=0$. While for every $k \neq 0$ the Bogoliubov vacuum corresponds to an even state (the absence of quasi-particles implies zero or two particles), the mode $k=0$ plays a special role. In fact, the correspondent Bogoliubov transformation reads

$$
\eta_{0}=\frac{1}{2}\left(1+\frac{h-1}{|h-1|}\right) c_{0}+\frac{i}{2}\left(1-\frac{h-1}{|h-1|}\right) c_{0}^{\dagger}
$$

with the important consequence that the quasi-particle vacuum corresponds to a zero-particle state for $h<1$ and to one-particle state for $h>1$. The presence or the absence of the particle in the $k=0$ mode changes the parity of the state. Thus, for $h>1$, the vacuum of $H^{-}$, because of its symmetry, does not belong to the set of eigenstates of $H$, while for $h<1$ it becomes an eigenstate of physical interest. Above the $h=1$, the odd state of lowest energy is obtained by adding one quasi-particle corresponding to the bottom of the energy band with energy $\Lambda_{\min }=2(h-1)$. This energy gap prevents the degeneracy even in the thermodynamic limit.

\section{A. Quantum phase transition and ground state factorization}

The change of symmetry of the vacuum of $\mathrm{H}^{-}$is the very cause of the phase transition in the thermodynamic limit. Indeed, on the macroscopic scale the sum over $k$ becomes an integral yielding $E_{0}^{+}=E_{0}^{-}$. Then, below the critical point $h_{C}=1$ the odd and the even lowest eigenstates are degenerate, and the Hamiltonian symmetry is spontaneously broken, while, for $h>h_{C}$, due to the existence of the energy gap $\Lambda_{\min }$, the ground state keeps its parity (even). For $h<h_{C}$, because of superselection rules, the system is necessarily found in symmetry-broken states.

As pointed out in Ref. [8], below the critical point there are two different regions where two-body correlation functions can decrease monotonically or oscillate as a function of the spin distance, depending on the Hamiltonian parameters. These regions are separate, in the $\{h, \gamma\}$ diagram, by the set of points satisfying $h_{F}^{2}+\gamma^{2}=1$. More recently, it has been shown that on this border line the ground state factorizes [23], i.e. it can be written as $\left|\Psi_{F}^{ \pm}\right\rangle=$ $\otimes_{l}\left|\Psi_{F, l}^{ \pm}\right\rangle$, with $\left|\Psi_{F, l}^{ \pm}\right\rangle=\left(\cos \alpha\left|\uparrow_{l}\right\rangle \pm \sin \alpha\left|\downarrow_{l}\right\rangle\right)$, where $\cos 2 \alpha=[(1-\gamma) /(1+\gamma)]^{1 / 2}$.

The existence of the factorizing field, originally derived by requiring only size-independent degeneracy between the lowest odd and even eigenvalues [3], can be studied within the general solution of the model. By analyzing lowest odd and even eigenvalues of $H$ in the symmetry broken region for finite $N$ as a function of the transverse field, we observe a series of $N / 2$ level crossings for $h=h_{i}$ (see Fig. 1). In correspondence of each $h_{i}$ the ground state changes its symmetry. The existence of such points has been discussed in Ref. [18] and more recently in Ref. [19], and is responsible for the magnetization jumps reported in Ref. [20]. In the thermodynamic limit, this kind of structure implies two different symmetry breaking mechanisms. For $0<h<h_{F}$, as $N \rightarrow \infty$, the set $\left\{h_{i}\right\}$ of the degeneracy points becomes a denumerable infinity, while for $h_{F}<h<1$ there is a the usual symmetry breaking due to the vanishing of the gap. An interesting problem would be to check whether this is the microscopic mechanism responsible for the qualitative change in the behavior of the correlation functions above and below $h_{F}$. 


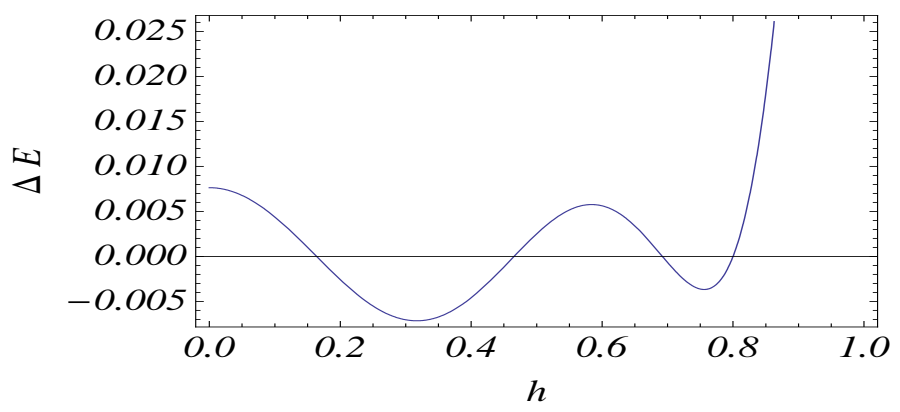

FIG. 1: Difference of energy between the lowest odd and even eigenvalues of $H$ as a function of the transverse field for a 8-spin chain. Given the anisotropy amplitude $\gamma=0.6$, the factorizing field is $h_{F}=0.8$. As predicted, we observe $N / 2$ level crossing point, the last of them being $h_{F}$.

While spontaneous symmetry breaking arises only for $N \rightarrow \infty$, it can be seen from the previous analysis (see also Ref. [19]) that at the factorizing point $h_{F}$ degeneracy appears for any $N$. It is simple to show that $E_{0}^{+}\left(h_{F}\right)=E_{0}^{-}\left(h_{F}\right)$ holds for any $N$, while the positions of all the other level crossing points $h_{i}$ change with $N$. Then, at the special field $h_{F}$, the Hamiltonian symmetry is broken independently of the system size, and any linear superposition of the two symmetric eigenstates $\left|\alpha^{ \pm}\right\rangle$( $\left|\alpha^{+}\right\rangle$for the even eigenstate and $\left|\alpha^{-}\right\rangle$for the odd eigenstate), is a possible eigenstate. Obviously, each one of the factorized states can be expressed as a linear combination of the two symmetric eigenstates

$$
\left|\Psi_{F}^{ \pm}\right\rangle=u_{+}\left|\alpha^{+}\right\rangle \pm u_{-}\left|\alpha^{-}\right\rangle
$$

with $u_{ \pm}=\left[\left(1+\cos ^{N} 2 \alpha\right) / 2\right]^{1 / 2}$. Notice that, for finite size systems, $\left|\Psi_{F}^{+}\right\rangle$and $\left|\Psi_{F}^{-}\right\rangle$are not orthogonal, while $\left\langle\Psi_{F}^{+} \mid \Psi_{F}^{-}\right\rangle=0$ in the thermodynamic limit.

\section{XXX MODEL}

The homogeneous (ferromagnetic) Heisenberg model is defined by the Hamiltonian

$$
H_{X X X}=-J \sum_{l=0}^{N-1}\left(\sigma_{l}^{x} \sigma_{l+1}^{x}+\sigma_{l}^{y} \sigma_{l+1}^{y}+\sigma_{l}^{z} \sigma_{l+1}^{z}\right),
$$

with the boundary condition $\sigma_{N}^{\epsilon}=\sigma_{0}^{\epsilon}$. The model has been solved using the Bethe ansatz [21]. As far as the ground state properties are concerned, a simple argument can be introduced to show that, for any number of spins, any factorized state $|\Phi(\theta, \phi)\rangle=\otimes_{l}[\cos \theta|\uparrow\rangle+\exp (i \phi) \sin \theta|\downarrow\rangle]$ minimizes the energy. Given the invariance of $H_{X X X}$ with respect to rotations of arbitrary amplitude $\beta$ around any direction $\hat{n} \mathcal{R}(\beta, \hat{n})=\prod_{l} \exp \left[i \beta \vec{\sigma}_{l} \cdot \hat{n}\right]$, due to $\left[H_{X X X}, \mathcal{R}(\beta, \hat{n})\right]=0$, we shall restrict the attention on the particular state $|\Phi(0,0)\rangle=|\uparrow, \uparrow, \ldots, \uparrow\rangle$. It can be immediately seen that $|\Phi(0,0)\rangle$ belongs to the ground state subspace of any of two-body terms of $H_{X X X}$, and then its energy represents the minimum achievable value. To make a link with the XY model, we could say that factorization point for the Heisenberg model corresponds to $h=0$.

In the absence of spontaneous symmetry breaking, i.e. for finite systems, and in the absence of external fields, the ground state belongs to an $(N+1)$-dimensional manifold, and can be expanded in the over-complete set of factorized states

$$
|\Phi\rangle=\frac{1}{\mathcal{N}^{\prime}} \int d \theta \int d \phi f(\theta, \phi)|\Phi(\theta, \phi)\rangle,
$$

where $f(\theta, \phi)$ is a weight function. The inner product between states pointing in different directions reads

$$
\left\langle\Phi\left(\theta^{\prime}, \phi^{\prime}\right) \mid \Phi(\theta, \phi)\right\rangle=\left[\cos \theta \cos \theta^{\prime}+e^{i\left(\phi-\phi^{\prime}\right)} \sin \theta \sin \theta^{\prime}\right]^{N} .
$$

Then, only in the thermodynamic limit we have a set of orthogonal states. Given the continuous $S U(2)$ symmetry of the model, spontaneous symmetry breaking implies that the system will select one direction out of all the possible choices in the $(\theta, \phi)$ space.

As we are interested in studying the problem of vanishing of peculiar quantum properties, we shall choose initial states with given symmetry properties, which, in the finite size limit, do exhibit those properties. 


\section{TRANSITION TO THE SCHRÖDINGER CAT REGIME}

According to the superposition principle, every linear combination of quantum states is allowed. On the other hand, it is well known that superposition cannot be observed on the macroscopic scale because of superselection, the most convincing argument being the Schrödinger cat paradox. Then, on this scale, all but a small set of states belonging the total Hilbert space are actually forbidden. This process, which leads to a diagonal form of the density operator in a preferred basis, implies the vanishing of the most peculiar of quantum properties: state interference.

In order to study the vanishing of state interference we analyze two different quantities: two-spin entanglement and the fluctuation properties of the order parameter $M_{x}=\left(\sum_{l} \sigma_{l}^{x}\right) / N$. Given a density matrix $\rho$, fluctuations statistics is associated to the generating function

$$
G_{\rho}(\lambda)=\operatorname{Tr}\left\{\rho e^{\frac{i \lambda}{N} \sum_{l} \sigma_{l}^{x}}\right\}
$$

which is the Fourier transform of the probability distribution function of $M_{x}$. When the system can be observed in $m$ states $\Psi_{1}, \Psi_{2}, \ldots \Psi_{m}$, whit related generating functions $G_{\Psi_{n}}(\lambda)$, quantum superposition effects appear if

$$
\Delta G=G_{\rho}(\lambda)-\frac{1}{m} \sum_{n=1}^{m} G_{\Psi_{n}}(\lambda) \neq 0 .
$$

We expect that in the symmetry broken regime $\lim _{N \rightarrow \infty} \Delta G=0$.

Similar considerations, carried out about entanglement properties, lead to establish that, when $N \rightarrow \infty$, only factorized states can be observed.

Even if superselection can be assumed as a principle, the size dependence of quantum interference effects will be related to the particular system observed. In the following, we find different decaying behaviors for the XY and the XXX models, which are caused by the difference in the symmetry of the two systems. We will start in both models by considering symmetric states (which are expected not to survive in the thermodynamic limit) and we shall study two-spin entanglement as a function of $N$. In fact, the existence of degenerate ground state manifolds for any $N$ allows to calculate coherence properties as a function of the size of the system.

For qubit systems, like spins, two-body entanglement can be measured through concurrence [22]. As shown in Ref. [23], for states which are invariant under the action of the parity operator, the concurrence $\mathcal{C}_{i j}$ of two spins at sites $i$ and $j$ is related to the quantum correlation functions by simple relations which will be used here. As pointed out in Ref. [19], for the XY model at the factorizing field the two-spin concurrence does not depend on the spin distance $|i-j|$. Similar arguments can be used also for the XXX chain. Since we are dealing with superpositions of ferromagnetic states, the entanglement will be of ferromagnetic kind as well. In this case,

$$
\mathcal{C}_{i j}=\frac{1}{2}\left|p_{1}-p_{2}\right|-p_{I I I}
$$

where $\left|p_{1}-p_{2}\right|$ is the average value of $|\uparrow \uparrow\rangle\langle\downarrow \downarrow|+| \downarrow \downarrow\rangle\langle\uparrow \uparrow|$, and $p_{I I I}$ is the average value of $|\uparrow \downarrow\rangle\langle\uparrow \downarrow|$.

\section{A. XY model}

Let us first consider the order parameter fluctuations for the symmetric states $\left|\alpha^{ \pm}\right\rangle$. These states could be obtained by starting with $h \neq h_{F}$. In this case the exact ground state would have definite parity. For instance, for $h>h_{F}$, the ground state is even. By lowering the field until the value $h_{F}$ is reached, the system is driven in $\left|\alpha^{+}\right\rangle$. The generating function is

$$
G\left(\lambda, \alpha^{ \pm}\right)=\frac{1}{4 u_{ \pm}^{2}}\left[G\left(\lambda, \Psi_{F}^{+}\right)+G\left(\lambda, \Psi_{F}^{-}\right)+\tilde{G}\left(\lambda, \Psi_{F}^{+}, \Psi_{F}^{-}\right)+\tilde{G}\left(\lambda, \Psi_{F}^{-}, \Psi_{F}^{+}\right)\right]
$$

where

$$
\tilde{G}\left(\lambda, \Psi_{F}^{ \pm}, \Psi_{F}^{\mp}\right)=\left\langle\Psi_{F}^{ \pm}\left|e^{\frac{i \lambda}{N} \sum_{l} \sigma_{l}^{x}}\right| \Psi_{F}^{\mp}\right\rangle
$$

It is easy to show that

$$
G\left(\lambda, \Psi_{F}^{ \pm}\right)=\left(\cos \frac{\lambda}{N} \pm i \sin \frac{\lambda}{N} \sin 2 \theta\right)^{N}
$$




$$
\tilde{G}\left(\lambda, \Psi_{F}^{ \pm}, \Psi_{F}^{\mp}\right)=\left(\cos \frac{\lambda}{N} \cos 2 \theta\right)^{N}
$$

Then, interference effects (manifested by the off diagonal elements) disappear exponentially with $N$.

As a second characterization, we study the concurrence for the symmetric states $\left|\alpha^{ \pm}\right\rangle$. This can be easily derived using the expression of $\left|\alpha^{ \pm}\right\rangle$in terms of $\left|\Psi_{F}^{ \pm}\right\rangle$. The result (see also Ref. [19]) is given by

$$
\mathcal{C}_{i j}\left(\alpha^{ \pm}\right)=(\cos 2 \alpha)^{N-2} \frac{\sin ^{2} 2 \alpha}{1 \pm(\cos 2 \alpha)^{N}},
$$

where the factor $(\cos 2 \theta)^{N-2}$ derives from the non-orthogonality of $\left|\Psi_{F}^{+}\right\rangle$and $\left|\Psi_{F}^{-}\right\rangle$and determines the speed of classicalization. In the macroscopic limit, $\mathcal{C}_{i j}\left(\alpha^{ \pm}\right)$vanishes as $[(1-\gamma) /(1+\gamma)]^{N / 2}$. Then, for every finite value of the anisotropy $\gamma$, entanglement decays exponentially with $N$. A small but finite anisotropy will enhance entanglement. Actually, the $\gamma=0$ limit implies a non-analytic change

\section{B. XXX model}

In analogy with the previous case, we introduce a state which is invariant under a given spin rotation. In particular, if we choose the state $\left|\Phi_{e}\right\rangle$ invariant under rotations about the $y$ axis $\exp \left[-i \theta \sum_{l} \sigma_{l}^{y}\right]\left|\Phi_{e}\right\rangle=\left|\Phi_{e}\right\rangle$, we have

$$
\left|\Phi_{e}\right\rangle=\frac{1}{\mathcal{N}} \int_{0}^{2 \pi} d \theta\left|\Phi_{\theta}\right\rangle
$$

where $\left|\Phi_{\theta}\right\rangle=\otimes_{l}\left|\Phi_{l}(\theta, 0)\right\rangle$, and where $\left|\Phi_{l}(\theta, 0)\right\rangle=\cos \theta\left|\uparrow_{l}\right\rangle+\sin \theta\left|\downarrow_{l}\right\rangle$. Requiring the normalization of $\left|\Phi_{e}\right\rangle$ implies $\int d \theta^{\prime} d \theta\left[\cos \left(\theta-\theta^{\prime}\right)\right]^{N}=\mathcal{N}^{2}$. It is easy to verify that $\left|\Phi_{e}\right\rangle$ is also an eigenstate of the parity operator. In fact, the integration over $\theta$ cancels, in the superposition, all terms with an odd number of down spins. Each $\left|\Phi_{\theta}\right\rangle$ would be the actual ground state in the presence of an external field directed along the direction $\theta$.

Let us analyze the order parameter. First, we calculate fluctuations for a given element of the ground state degeneracy manifold, obtaining

$$
G_{\Phi_{\theta}}(\lambda)=\frac{1}{\mathcal{N}^{2}}\left(\cos \frac{\lambda}{N}+i \sin \frac{\lambda}{N} \sin 2 \theta\right)^{N} .
$$

For large $N$ we see that $G_{\Phi_{\theta}}(\lambda) \simeq \exp (i \lambda \sin 2 \theta)$. This is the typical expression of the generating function of a non fluctuating quantity. Its Fourier transform, which is the probability distribution function of the order parameter is indeed, for any $\theta$, a Dirac's delta distribution around $\sin 2 \theta$.

Furthermore, in the superposition state we have

$$
G_{\Phi_{e}}(\lambda)=\frac{1}{\mathcal{N}^{2}} \int d \theta^{\prime} d \theta\left[g\left(\lambda, \theta, \theta^{\prime}\right)\right]^{N},
$$

where

$$
g\left(\lambda, \theta, \theta^{\prime}\right)=\cos \frac{\lambda}{N} \cos \left(\theta-\theta^{\prime}\right)+i \sin \frac{\lambda}{N} \sin \left(\theta+\theta^{\prime}\right) .
$$

As $N$ gets large, the vanishing of interference is observed. In the large $N$ limit, the steepest descent method gives

$$
\Delta G_{\Phi_{e}}(\lambda)=\int d \theta G_{\Phi_{\theta}}(\lambda)\left[\exp \left(\frac{\lambda^{2} \cos 2 \theta}{2 N}\right)-1\right]
$$

implying

$$
\Delta G_{\Phi_{e}}(\lambda) \sim 1 / N
$$

in the asymptotic regime.

As far as the two-spin concurrence is concerned, it is straightforward to find

$$
\left|p_{1}-p_{2}\right|=\frac{1}{\mathcal{N}^{2}} \int d \theta d \theta^{\prime}\left[\cos ^{2} \theta \sin ^{2} \theta^{\prime}+\cos ^{2} \theta^{\prime} \sin ^{2} \theta\right]\left[\cos \left(\theta-\theta^{\prime}\right)\right]^{N-2},
$$




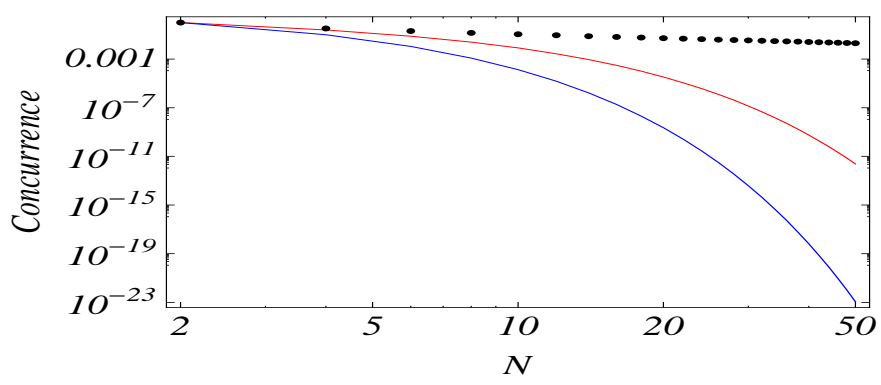

FIG. 2: Two-spin concurrence as a function of the system size $N$ for the XXX model (dots) and for the XY model (continuous lines). In this latter case, we considered two different anisotropy parameters: the red line represents $\gamma=0.5$, and the blue line corresponds to $\gamma=0.8$.

and

$$
p_{I I I}=\int d \theta d \theta^{\prime} \cos \theta \cos \theta^{\prime} \sin \theta \sin \theta^{\prime}\left[\cos \left(\theta-\theta^{\prime}\right)\right]^{N-2},
$$

yielding

$$
\mathcal{C}_{i j}\left(\Phi_{e}\right)=\frac{1}{2} \frac{\int d \theta d \theta^{\prime}\left[\tan \left(\theta-\theta^{\prime}\right)\right]^{2}\left[\cos \left(\theta-\theta^{\prime}\right)\right]^{N}}{\int d \theta d \theta^{\prime}\left[\cos \left(\theta-\theta^{\prime}\right)\right]^{N}} .
$$

This result is very simple for $N$ even. In that case one gets

$$
\mathcal{C}_{i j}^{N} \text { even }\left(\Phi_{e}\right)=\frac{1}{2(N-1)}
$$

This result can be understood taking into account that, given Eq. (14), the inner product between $|\Phi(\theta, 0)\rangle$ and $\left|\Phi\left(\theta^{\prime}, 0\right)\right\rangle$ vanishes exponentially with $N$. This allows to evaluate integrals, in the large $N$ regime, by means of the steepest descent method. It is clear that, when $N$ gets large, $\left[\cos \left(\theta-\theta^{\prime}\right)\right]^{N}$ is different from zero only for $\left(\theta-\theta^{\prime}\right)$ $\simeq 0$. Expanding all terms around this value, the concurrence is well approximated by the ratio between two Gaussian integrals

$$
\mathcal{C}_{i j}\left(\Phi_{e}\right) \simeq \frac{1}{2} \frac{\int_{-\infty}^{\infty} x^{2} \exp \left(-\frac{N x^{2}}{2}\right) d x}{\int_{-\infty}^{\infty} \exp \left(-\frac{N x^{2}}{2}\right) d x},
$$

which eventually gives $\mathcal{C}_{i j}\left(\Phi_{e}\right) \simeq 1 / 2 N$ in the asymptotic regime.

\section{DISCUSSION}

We tackled the problem of describing how quantum coherence effects vanish as the system size becomes macroscopic. Even if this phenomenon is expected to appear in generic systems, two different symmetry-broken model have been considered when exact and analytic treatment are possible. In the first one (the XY model in a transverse field), because of the discrete symmetry, the ground state spans a two-dimensional manifold. As for the Heisenberg model, the dimension of the manifold grows with $N$, eventually reaching a dense structure. In Fig. 2 we plot the behavior of the concurrence $\mathcal{C}_{i j}$ for the two models. In the case of the XY chain, we used also different values of the anisotropy . A necessary step to determine local quantities, like two-spin entanglement or magnetization, is the introduction of the reduced density matrix. Given the peculiar structure of the factorized states we have considered here, calculating the reduced density matrix requires the computation of inner products between states defined on $(N-m)$ (where $m$ is a finite number) spin subspaces) aligned along different directions. Both for the XY and for the isotropic model these quantities vanish exponentially in the large $N$ limit, destroying in such a way the quantum interference between different components. However, since the XXX model has a continuous symmetry, the ground state manifold is continuous as well, and all matrix elements are integrated. The integration implies a reduction in the decoherence rate, which turns out to be linear in $1 / N$. 
In this paper we considered two exactly solvable models, and studied how superselection tends to destroy their quantum properties. A typical tool used to study problems whose solution is not known is the mean-field approximation, that, in fact, consists in the introduction of "product states" with the same aspect of those described in this paper. For example, in the BCS theory of superconductivity the solution introduced is factorized in the space of the modes $k$. Since in the finite-size case the symmetry is expected to be conserved, while the mean-field states are widely unsymmetrical, the linear superposition of degenerate states is a way to restore it. Once the thermodynamic limit is performed, all the considerations made in this paper apply. Then, we can conclude that our results apply not only to the models explicitly studied, but they could used, in the limit of validity of the mean-field theory, in all systems belonging to the classes of symmetry discussed.

\section{Acknowledgments}

The authors wish to acknowledge S. Paganelli for useful discussions. GLG acknowledges the Juan de la Cierva fellowship of the Spanish Ministry of Science and Innovation.

[1] D. Giulini, E. Joos, C. Kiefer, J. Kupsch, I. O. Stamatescu and H. D. Zeh Decoherence and the appearence of a Classical World in Quantum Theory (Springer, Berlin) (1996).

[2] W. H. Zurek, Rev. Mod. Phys. 75715 (2003).

[3] J. Kurmann, H. Thomas and G. Muller, Physica (Amsterdam) 112A, 235 (1982).

[4] O. F. Syljuåsen, Phys. Rev. A 68, 060301(R) (2003).

[5] A. Osterloh, G. Palacios, an S. Montangero, Phys. Rev. Lett. 97, 257201 (2006)

[6] S. Katsura, Phys. Rev. 127, 1508 (1962).

[7] E. Lieb, T. Schultz and D. Mattis, Ann. Phys. (N.Y.) 16, 407 (1961).

[8] E. Barouch and B. M. McCoy, Phys. Rev. A, 3, 786 (1971).

[9] T. J. Osborne and M. A. Nielsen, Phys. Rev. A 66, 032110 (2002).

[10] A. Osterloh, L. Amico, G. Falci, and R. Fazio, Nature (London) 416, 608 (2002).

[11] G. Vidal, J. I. Latorre, E. Rico, and A. Kitaev, Phys. Rev. Lett. 90, 227902 (2003).

[12] L. Amico, R. Fazio, A. Osterloh, and V. Vedral, Rev. Mod. Phys. 80, 517 (2008)

[13] L. Amico, F. Baroni, A. Fubini, D. Patanè, V. Tognetti, and P. Verrucchi, Phys. Rev. 1 74, 022322 (2006).

[14] F. Baroni, A. Fubini, V. Tognetti, and P. Verrucchi, J. Phys. A: Math. Theor. 40, 9845 (2007).

[15] S. M. Giampaolo, G. Adesso, and F. Illuminati, Phys. Rev. Lett. 100, 197201 (2008).

[16] G. L. Giorgi, Phys. Rev. B 79, 060405 (2009).

[17] V. Vedral, Nature (London) 453, 1004 (2008).

[18] C. Hoeger, G. von Gehlen, and V. Rittenberg, J. Phys. A 18, 1813 (1985).

[19] R. Rossignoli, N. Canosa, and J. M. Matera, Phys. Rev. A 77, 052322 (2008).

[20] J. Kurmann, G. Müller, H. Thomas, M. W. Puga, and H. Beck, J. Appl. Phys. 52, 1968 (1981).

[21] H. A. Bethe, Z. Phys. 71, 205 (1931).

[22] W. K. Wootters, Phys. Rev. Lett. 80, 2245 (1998).

[23] A. Fubini, T. Roscilde, V. Tognetti, M. Tusa, and P. Verrucchi, Eur. Phys. J. D 38, 563 (2006). 